\title{
Recognizing Structural Patterns on Graphs for the Efficient Computation of \#2SAT
}

\author{
Guillermo De Ita, Pedro Bello, and Meliza Contreras \\ Benemérita Universidad Autónoma de Puebla, Faculty of Computer Science \\ Av. San Claudio y 14 sur, Puebla, México \\ \{deita, pbello, mcontreras\}@cs.buap.mx
}

\begin{abstract}
To count models for two conjunctive forms (\#2SAT problem) is a classic \#P problem. We determine different structural patterns on the underlying graph of a $2-\mathrm{CF} F$ allowing the efficient computation of \#2SAT $(F)$.

We show that if the constrained graph of a formula is acyclic or the cycles on the graph can be arranged as independent and embedded cycles, then the number of models of $F$ can be counted efficiently.
\end{abstract}

Keywords: \#SAT Problem, Counting models, Structural Patterns, Graph Topologies.

\section{Introduction}

\#SAT is of special concern to Artificial Intelligence (AI), and it has a direct relationship to Automated Theorem Proving, as well as to approximate reasoning 3419 .

The problem of counting models for a Boolean formula (\#SAT problem) can be reduced to several different problems in approximate reasoning. For example, for estimating the degree of reliability in a communication network, computing degree of belief in propositional theories, for the generation of explanations to propositional queries, in Bayesian inference, in a truth maintenance systems, for repairing inconsistent databases 11315/910. The previous problems come from several AI applications such as planning, expert systems, approximate reasoning, etc.

\#SAT is at least as hard as the SAT problem, but in many cases, even when SAT is solved in polynomial time, no computationally efficient method is known for \#SAT. For example, 2-SAT problem (SAT restricted to consider $(\leq 2)$-CF's), it can be solved in linear time. However, the corresponding counting problem \#2-SAT is a \#P-complete problem. Earlier works on \#2-SAT include papers by Dubois [6], Zhang [11 and Littman [8. More recently, new upper bounds for exact deterministic algorithms for \#2-SAT have been found by Dahllöf [2], Fürer [7, Angelsmark [1 and Jonsson [2]. And given that \#2SAT is a \#P-complete problem, all the above proposals are part of the class of exponential algorithms.

The maximum polynomial class recognized for \#2SAT is the class $(\leq 2,2 \mu)$ $\mathrm{CF}$ (conjunction of binary or unary clauses where each variable appears twice at 
most) 910. Here, we extend such class for considering the topological structure of the undirected graph induced by the restrictions (clauses) of the formula. We extend here some of the procedures presented in [5.4] for the \#2-SAT problem and show how to apply them to compute the number of models in a propositional theory. Furthermore, we show different structural patterns on the constrained graph of the formula which allow the efficient computation of the number of models for some classes of 2-CF's.

\section{Notation}

Let $X=\left\{x_{1}, \ldots, x_{n}\right\}$ be a set of $n$ Boolean variables. A literal is either a variable $x_{i}$ or a negated variable $\overline{x_{i}}$. As usual, for each $x_{i} \in X, x_{i}^{0}=x_{i}$ and $x_{i}^{1}=x_{i}$. A clause is a disjunction of different literals (sometimes, we also consider a clause as a set of literals). For $k \in N$, a $k$-clause is a clause consisting of exactly $k$ literals and, a $(\leq k)$-clause is a clause with at most $k$ literals. A variable $x \in X$ appears in a clause $c$ if either $x$ or $\bar{x}$ is an element of c.

A Conjunctive Form (CF) $F$ is a conjunction of clauses (we also consider a $\mathrm{CF}$ as a set of clauses). We say that $F$ is a positive monotone $\mathrm{CF}$ if all of its variables appear in unnegated form. $\mathrm{A} k$-CF is a $\mathrm{CF}$ containing only $k$-clauses and, $(\leq k)$ - CF denotes a CF containing clauses with at most $k$ literals. A $k \mu-$ $\mathrm{CF}$ is a formula in which no variable occurs more than $k$ times. A $(k, j \mu)-\mathrm{CF}$ $((\leq k, j \mu)-\mathrm{CF})$ is a $k$-CF $((\leq k)-\mathrm{CF})$ such that each variable appears no more than $j$ times.

We use $\nu(X)$ to express the set of variables involved in the object $X$, where $X$ could be a literal, a clause or a Boolean formula. For instance, for the clause $c=\left\{\overline{x_{1}}, x_{2}\right\}, \nu(c)=\left\{x_{1}, x_{2}\right\}$. And $\operatorname{Lit}(F)$ is the set of literals which appear in a CF $F$, i.e. if $X=\nu(F)$, then $\operatorname{Lit}(F)=X \cup \bar{X}=\left\{x_{1}, \overline{x_{1}}, \ldots, x_{n}, \overline{x_{n}}\right\}$. We also denote $\{1,2, \ldots, n\}$ by $[[n]]$.

An assignment $s$ for $F$ is a Boolean function $s: \nu(F) \rightarrow\{0,1\}$. An assignment can be also considered as a set of non complementary pairs of literals. If $l \in s$, being $s$ an assignment, then $s$ turns $l$ true and $\bar{l}$ false. Considering a clause $c$ and assignment $s$ as a set of literals, $c$ is satisfied by $s$ if and only if $c \cap s \neq 0$, and if for all $l \in c, \bar{l} \in s$ then $s$ falsifies $c$. If $F_{1} \subset F$ is a formula consisting of some clauses of $F$, then $\nu(F 1) \subset \nu(F)$, and an assignment over $\nu\left(F_{1}\right)$ is a partial assignment over $\nu(F)$. Assuming $n=|\nu(F)|$ and $n_{1}=\left|\nu\left(F_{1}\right)\right|$, any assignment over $\nu\left(F_{1}\right)$ has $2^{n-n_{1}}$ extensions as assignments over $\nu(F)$.

Let $F$ be a Boolean formula in Conjunctive Form $(\mathrm{CF}), F$ is satisfied by an assignment $s$ if each clause in $F$ is satisfied by $s . F$ is contradicted by $s$ if any clause in $F$ is contradicted by $s$. A model of $F$ is an assignment for $\nu(F)$ that satisfies $F$. Given $F$ a CF, the SAT problem consists of determining if $F$ has a model. The \#SAT problem consists of counting the number of models of $F$ defined over $\nu(F)$. \#2-SAT denotes \#SAT for formulas in 2-CF. 


\section{Computing \#2SAT for Acyclic Formulas}

Let $F$ be a 2-CF $F$, its signed constrained undirected graph is denoted by $G_{F}=$ $(V(F), E(F))$, with $V(F)=\nu(F)$ and $E(F)=\{\{\nu(x), \nu(y)\}:\{x, y\} \in F\}$, that is, the vertices of $G_{F}$ are the variables of $F$, and for each clause $\{x, y\}$ in $F$ there is an edge $\{\nu(x), \nu(y)\} \in E(F)$. Each edge $c=\{\nu(x), \nu(y)\} \in E$ is associated with an ordered pair $\left(s_{1}, s_{2}\right)$ of signs, assigned as labels of the edge connecting the variables appearing in the clause. The signs $s_{1}$ and $s_{2}$ are related to the signs of the literals $x$ and $y$ respectively. For example, the clause $\{\bar{x} \vee y\}$ determines the labelled edge: " $x-\frac{-+}{} y$ " which is equivalent to the edge " $y \stackrel{+-}{ } x "$.

A graph with labelled edges on a set $S$ is a pair $(G, \psi)$, where $G=(V, E)$ is a graph, and $\varphi$ is a function with domain $E$ and range $S . \psi(e)$ is called the label of the edge $e \in E$. Let $S=\{+,-\}$ be a set of signs . Let $G=(V, E, \psi)$ be a signed graph with labelled edges on $S \times S$. Let $x$ and $y$ be nodes in $V$. If $e=\{x, y\}$ is an edge and $\psi(e)=\left(s, s^{\prime}\right)$, then $s\left(s^{\prime}\right)$ is called the adjacent sign to $x(y)$. We say that a $2-\mathrm{CF} F$ is a path, cycle, or a tree if its signed constrained graph $G_{F}$ is a path, cycle, or a tree, respectively.

\subsection{If the 2-CF Represents a Path}

If $G_{F}$ is a path, then $F=\left\{C_{1}, C_{2}, \ldots, C_{m}\right\}=\left\{\left\{x_{1}^{\epsilon_{1}}, x_{2}^{\delta_{1}}\right\},\left\{x_{2}^{\epsilon_{1}}, x_{3}^{\delta_{1}}\right\}, \ldots,\left\{x_{m}^{\epsilon_{m}}\right.\right.$, $\left.\left.x_{m+1}^{\delta_{m}}\right\}\right\}$, where $\delta_{i}, \epsilon_{i} \in\{0,1\}, i \in \llbracket m \rrbracket$. Let $f_{i}$ be a family of clauses of the formula $F$, built as follows: $f_{1}=\emptyset ; f_{i}=\left\{C_{j}\right\}_{j<i}, i \in \llbracket m \rrbracket$. Notice that $n=$ $|v(F)|=m+1, f_{i} \subset f_{i+1}, i \in \llbracket m-1 \rrbracket$. Let $S A T\left(f_{i}\right)=\left\{s: s\right.$ satisfies $\left.f_{i}\right\}, A_{i}=$ $\left\{s \in S A T\left(f_{i}\right): x_{i} \in s\right\}, B_{i}=\left\{s \in S A T\left(f_{i}\right): \bar{x}_{i} \in s\right\}$. Let $\alpha_{i}=\left|A_{i}\right| ; \beta_{i}=\left|B_{i}\right|$ and $\mu_{i}=\left|S A T\left(f_{i}\right)\right|=\alpha_{i}+\beta_{i}$.

For every node $x \in G_{F}$ a pair $\left(\alpha_{x}, \beta_{x}\right)$ is computed, where $\alpha_{x}$ indicates how many times the variable $x$ is 'true' and $\beta_{x}$ indicates the number of times that the variable $x$ can take value 'false' into the set of models of $F$. The first pair is $\left(\alpha_{1}, \beta_{1}\right)=(1,1)$ since $x_{1}$ can be true or false in order to satisfy $f_{1}$. The pairs $\left(\alpha_{x}, \beta_{x}\right)$ associated to each node $x_{i}, i=2, \ldots, m$ are computed according to the signs $\left(\epsilon_{i}, \delta_{i}\right)$ of the literals in the clause $c_{i}$ by the following recurrence equation:

$$
\left(\alpha_{i}, \beta_{i}\right)= \begin{cases}\left(\beta_{i-1}, \alpha_{i-1}+\beta_{i-1}\right) & \text { if }\left(\epsilon_{i}, \delta_{i}\right)=(-,-) \\ \left(\alpha_{i-1}+\beta_{i-1}, \beta_{i-1}\right) & \text { if }\left(\epsilon_{i}, \delta_{i}\right)=(-,+) \\ \left(\alpha_{i-1}, \alpha_{i-1}+\beta_{i-1}\right) & \text { if }\left(\epsilon_{i}, \delta_{i}\right)=(+,-) \\ \left(\alpha_{i-1}+\beta_{i-1}, \alpha_{i-1}\right. & \text { if }\left(\epsilon_{i}, \delta_{i}\right)=(+,+)\end{cases}
$$

Note that, as $F=f_{m}$ then $\# S A T(F)=\mu_{m}=\alpha_{m}+\beta_{m}$. We denote with $\rightarrow$ the application of one of the four rules of the recurrence (1).

Example 1. Let $F=\left\{\left(x_{1}, x_{2}\right),\left(\bar{x}_{2}, \bar{x}_{3}\right),\left(\bar{x}_{3}, \bar{x}_{4}\right),\left(x_{4}, \bar{x}_{5}\right),\left(\bar{x}_{5}, x_{6}\right)\right\}$ be a path. The series $\left(\alpha_{i}, \beta_{i}\right), i \in \llbracket 6 \rrbracket$, is computed as: $\left(\alpha_{1}, \beta_{1}\right)=(1,1) \rightarrow\left(\alpha_{2}, \beta_{2}\right)=(2,1)$ since $\left(\epsilon_{1}, \delta_{1}\right)=(1,1)$, and the rule 4 has to be applied. In general, applying the corresponding rule of the recurrence (11) according to the signs expressed by $\left(\epsilon_{i}, \delta_{i}\right), i=3, \ldots, 6$, we have $(2,1) \rightarrow(1,3) \rightarrow(3,4) \rightarrow(3,7) \rightarrow\left(\alpha_{6}, \beta_{6}\right)=(10,7)$, and then, $\# S A T(F)=\mu_{6}=\alpha_{6}+\beta_{6}=10+7=17$. 


\subsection{If the 2-CF Represents a Tree}

Let $F$ be a $2-C F$ where its associated constrained graph $G_{F}$ is a tree. We denote with $\left(\alpha_{v}, \beta_{v}\right)$ the pair associated with the node $v\left(v \in G_{F}\right)$. We compute $\# S A T(F)$ while we are traversing by $G_{F}$ in post-order.

\section{Algorithm Count_Models_for_trees $\left(G_{F}\right)$}

Input: $G_{F}$ - a tree graph.

Output: The number of models of $F$

Procedure:

Traversing $G_{F}$ in post-order, and when a node $v \in G_{F}$ is left, assign:

1. $\left(\alpha_{v}, \beta_{v}\right)=(1,1)$ if $v$ is a leaf node in $G_{F}$.

2. If $v$ is a parent node with a list of child nodes associated, i.e., $u_{1}, u_{2}, \ldots, u_{k}$ are the child nodes of $v$, as we have already visited all child nodes, then each pair $\left(\alpha_{u_{j}}, \beta_{u_{j}}\right) j=1, \ldots, k$ has been determined based on recurrence (1). Then, let $\alpha_{v}=\prod_{j=1}^{k} \alpha_{v_{j}}$ and $\beta_{v}=\prod_{j=1}^{k} \beta_{v_{j}}$. Notice that this step includes the case when $v$ has just one child node.

3. If $v$ is the root node of $G_{F}$ then $\operatorname{return}\left(\alpha_{v}+\beta_{v}\right)$.

This procedure returns the number of models for $F$ in time $O(n+m)$ which is the necessary time for traversing $G_{F}$ in post-order.

Example 2. If $F=\left\{\left(x_{1}, x_{2}\right),\left(x_{2}, x_{3}\right),\left(x_{2}, x_{4}\right),\left(x_{2}, x_{5}\right),\left(x_{4}, x_{6}\right),\left(x_{6}, x_{7}\right)\right.$, $\left.\left(x_{6}, x_{8}\right)\right\}$ is a monotone 2-CF, we consider the post-order search starting in the node $x_{1}$. The number of models at each level of the tree is shown in Figure 1. The procedure Count_Models_for_trees returns for $\alpha_{x_{1}}=41, \beta_{x_{1}}=36$ and the total number of models is: \#SAT(F)=41+36=77.

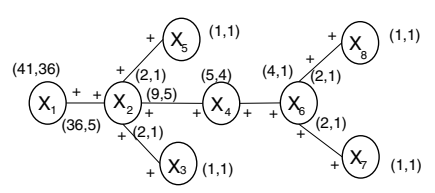

Fig. 1. Counting models over a tree

\section{Processing 2-CF's Containing Cycles}

Let $G_{F}$ be a simple cycle with $m$ nodes, that is, all the variables in $v(F)$ appear twice, $|V|=m=n=|E|$. Ordering the clauses in $F$ in such a way that $\left|v\left(c_{i}\right) \cap v\left(c_{i+1}\right)\right|=1$, and $c_{i_{1}}=c_{i_{2}}$ whenever $i_{1} \equiv i_{2} \bmod m$, hence $x_{1}=x_{m}$, then $F=\left\{c_{i}=\left\{x_{i-1}^{\epsilon_{i}}, x_{i}^{\delta_{i}}\right\}\right\}_{i=1}^{m}$, where $\delta_{i}, \epsilon_{i} \in\{0,1\}$. Decomposing $F$ as $F=$ $F^{\prime} \cup c_{m}$, where $F^{\prime}=\left\{c_{1}, \ldots, c_{m-1}\right\}$ is a path and $c_{m}=\left(x_{m-1}^{\epsilon_{m}}, x_{1}^{\delta_{m}}\right)$ is the edge which conforms with $G_{F^{\prime}}$ the simple cycle: $x_{1}, x_{2}, \ldots, x_{m-1}, x_{1}$. We will call to 
$G_{F^{\prime}}$ the internal path of the cycle and to $c_{m}$ the back clause of the cycle. We can apply the linear procedure described above in equation 1 for computing \#SAT $\left(F^{\prime}\right)$.

Every model of $F^{\prime}$ had determined logical values for the variables: $x_{m-1}$ and $x_{1}$ since those variables appear in $v\left(F^{\prime}\right)$. Any model $s$ of $F^{\prime}$ satisfies $c_{m}$ if and only if $\left(x_{m-1}^{1-\epsilon_{m}} \notin s\right.$ and $\left.x_{m}^{1-\delta_{m}} \notin s\right)$, that is, $S A T\left(F^{\prime} \cup c_{m}\right) \subseteq S A T\left(F^{\prime}\right)$, and $S A T\left(F^{\prime} \cup c_{m}\right)=S A T\left(F^{\prime}\right)-\left\{s \in S A T\left(F^{\prime}\right): s\right.$ falsifies $\left.c_{m}\right\}$. Let $X=F^{\prime} \cup$ $\left\{\left(x_{m-1}^{1-\epsilon_{m}}\right) \wedge\left(x_{m}^{1-\delta_{m}}\right)\right\}$, then $\# S A T(X)$ is computed as a path with two unitary clauses:

$\# S A T(F)=\# S A T\left(F^{\prime} \wedge C_{m}\right)=\# S A T\left(F^{\prime}\right)-\# S A T\left(F^{\prime} \wedge\left(x_{m-1}^{1-\epsilon_{m}}\right) \wedge\left(x_{m}^{1-\delta_{m}}\right)\right)$

Example 1. Let $\Sigma=\left\{c_{i}\right\}_{i=1}^{6}=\left\{\left\{x_{1}, x_{2}\right\},\left\{x_{2}, x_{3}\right\},\left\{x_{3}, x_{4}\right\},\left\{x_{4}, x_{5}\right\},\left\{x_{5}, x_{6}\right\}\right.$, $\left.\left\{x_{6}, x_{1}\right\}\right\}$ be a monotone $2-\mathrm{CF}$ which represents a cycle: $G_{\Sigma}=(V, E)$. Let $G^{\prime}=$ $\left(V, E^{\prime}\right)$ where $E=E^{\prime} \cup\left\{c_{6}\right\}$, that is, the new graph $G^{\prime}$ is $\Sigma$ minus the edge $c_{6}$. Applying equation ( 2), we have that \#SAT( $\Sigma)=\# S A T\left(F^{\prime}\right)-\# S A T\left(F^{\prime} \wedge\right.$ $\left.\bar{x}_{6} \wedge \bar{x}_{1}\right)=21-3=18$. This example is illustrated in figure 2 .

When we count models over any constrained graph $G_{F}$, we use computing threads. A computing thread is a sequence of pairs $\left(\alpha_{i}, \beta_{i}\right), i=1, \ldots, m$ used for computing the number of models over a path of $m$ nodes. A main thread, denoted by $L_{p}$, is associated to a spanning tree of $G_{F}$, this thread is always active until the process of counting finishes completely. While the thread used for computing the pair associated with $\# S A T\left(F^{\prime} \wedge\left(x_{m-1}^{1-\epsilon_{m}}\right) \wedge\left(x_{m}^{1-\delta_{m}}\right)\right)$ is denoted by $L_{e}$.

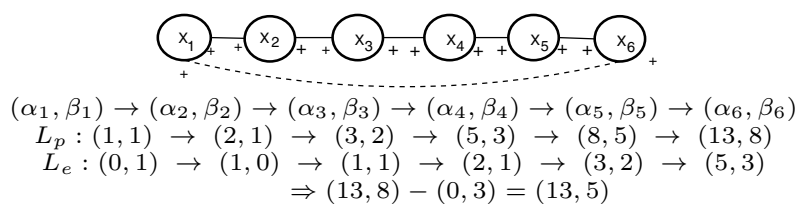

Fig. 2. Computing \#SAT(F) when $G_{F}$ is a cycle

\subsection{Cycles on Alternating Signed Paths}

Let $G_{F}=(V, E,\{+,-\})$ be a signed connected graph of an input formula $F$ in 2-CF. Let $v_{r}$ be the node of minimum degree in $G_{F}$ which is chosen to start a depth-first search. We obtain a spanning tree $T_{G}$ with $v_{r}$ as the root node and a set of fundamental cycles $C=\left\{C_{1}, C_{2}, \ldots, C_{k}\right\}$, and where each back edge $c_{i} \in E$ marks the beginning and the end of a fundamental cycle $C_{i} \in C$. 
The edges in $T_{G}$ are called tree edges. A back edge $e \in E$ is an edge of $G_{F}$ which is not part of the spanning tree $T_{G}$ but $e$ is incident to two nodes of $T_{G}$. Each back edge holds the maximum path contained in the fundamental cycle which is part of. We will call to such maximum path, the internal path of a fundamental cycle. Given any pair of fundamental cycles $C_{i}$ and $C_{j}$ in $G_{F}$, if $C_{i}$ and $C_{j}$ share edges, we call them intersecting cycles; otherwise, they are called independent cycles.

In some cases, the value $\# \operatorname{SAT}\left(C_{i}\right)$ for a fundamental cycle $C_{i} \in G_{F}$ can be computed previously to the computation of the total graph $G_{F}$ in order to determine if the cycle $C_{i}$ can be reduced to a path or any other simple structure. For example, let us assume a cycle $C_{i}$ whose internal path is formed by nodes with alternating signs on its edges, while the signs on the back edge $e$ determine the different cases to analyze. Let us order the nodes into the internal path of the cycle as: $x_{1}-x_{2}-\ldots-x_{k}$, and we consider to $x_{1}$ as the initial node and $x_{k}$ as the final node of the cycle. Notice that $e=\left\{x_{1}, x_{k}\right\}$ is the back edge.

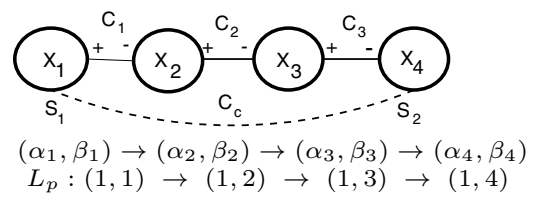

Fig. 3. $G_{F}$ and the computing over the path

Figure 3 shows a cycle with an internal path whose nodes have alternating signs. Let $\psi(e)=\left(a, b^{\prime}\right)$ be the signs on the back edge. Assuming that the variable $x_{1}$ appears only with sign $a$ and the variable $x_{k}$ appears only with sign $b$, that means that the signs of the back edge coincides with the signs of its endpoints in the internal path. For this case, the back edge (its corresponding clause) can be eliminated from the cycle because the final pair obtained in the secondary thread $L_{e}$ is $(0,0)$, as it is shown in figure 4

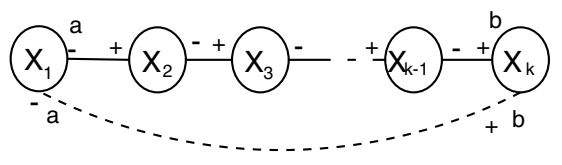

$$
\begin{aligned}
& (\alpha, \beta) \rightarrow(\alpha+\beta, \beta) \rightarrow(\alpha+2 \beta, \beta) \ldots \rightarrow(\alpha+k \cdot \beta, \beta) \Rightarrow(\alpha+k \cdot \beta, \beta) \\
& (\alpha, 0) \rightarrow(\alpha, 0) \quad \rightarrow(\alpha, 0) \quad \ldots \rightarrow(\alpha, 0) \quad \Rightarrow-(0,0)
\end{aligned}
$$

Fig. 4. When a back edge does not substract models to the path

The previous example shows that pre-processing the cycles appearing in a current constrained graph is relevant. In some cases, the clause corresponding 
with the back edge can be eliminated or the value \#SAT( $\left.C_{i}\right)$ for a cycle $C_{i}$ can be computed using symbolic values without knowing the real values of the pairs $\left(\alpha_{j}, \beta_{j}\right)$ on the internal path of the cycle, as it is shown in figure 5 .

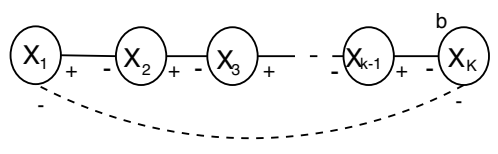

$$
\begin{aligned}
& (\alpha, \beta) \rightarrow(\alpha, \alpha+\beta) \rightarrow \ldots \rightarrow(\alpha, k \cdot \alpha+\beta) \Rightarrow(\alpha, k \cdot \alpha+\beta)-(\alpha, 0)=(0, k \cdot \alpha+\beta) \\
& (\alpha, 0) \rightarrow(\alpha, \alpha) \rightarrow \ldots \rightarrow(\alpha, k \cdot \alpha) \Rightarrow-(\alpha, 0)
\end{aligned}
$$

Fig. 5. Computing \#SAT $\left(C_{i}\right)$ using symbolic values on the pairs $\left(\alpha_{j}, \beta_{j}\right)$

\section{$5 \quad$ Processing Embedded Cycles}

Let $G_{F}=(V, E)$ be a connected constrained graph of a 2-CF $F$. Given two intersecting plane cycles $C_{i}, C_{j}$ of a graph, $C_{i}$ is embedded into $C_{j}$, if

a) $V\left(C_{i}\right) \subset V\left(C_{j}\right)$ : the set of nodes of $C_{i}$ is a subset of the nodes of $C_{j}$.

b) $\left|E\left(C_{i}\right)-E\left(C_{j}\right)\right|=1$ : there is only one edge from $C_{i}$ which is not edge of $C_{j}$. In this case, $C_{i}$ is an internal embedded cycle of $C_{j}$ and $C_{j}$ is an external embedded cycle of $C_{i}$.

Let us consider a graph $G_{F}$ formed by a set $D=\left(C_{1}, C_{2}, \ldots, C_{k}\right)$ of embedded cycles, such that $C_{i}$ is embedded in $C_{i+1}, i=1, \ldots, k-1 . C_{1}$ is the most internal embedded cycle and $C_{k}$ is the most external cycle of $D$. For processing this class of graphs, we determine a processing order given by traversing the graph from the most internal to the most external embedded cycle.

For a graph $G_{F}$ formed by a set of embedded cycles, $L p$ will be associated with the path formed by the nodes of $G_{F}$. Three computing threads are used for processing a current cycle, and for processing all the set of embedded cycles we require at most six computing threads.

\section{Case 1: Processing the Most Internal Embedded Cycle}

Let $e_{b}=\left\{v_{s}, v_{f}\right\}$ be the back edge which embraces the most internal cycle $C_{1}$ of $G_{F}$. We use three computing threads with initial values: $\left(\alpha_{s}^{1}, \beta_{s}^{1}\right)=(1,1)$, $\left(\alpha_{s}^{2}, \beta_{s}^{2}\right)=(1,0)$ - this thread carry on the number of models of $C_{1}$ where the

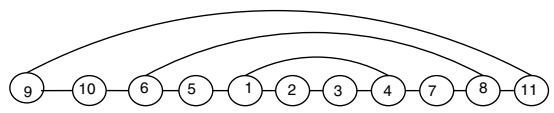

Fig. 6. An initial graph $G_{F}$ with embedded cycles 
variable $x_{s}$ does not appear, and $\left(\alpha_{s}^{3}, \beta_{s}^{3}\right)=(0,1)$ - this thread carry on the number of models of $C_{1}$ where the first variable $x_{0}$ appears.

We traverse by the internal path of $C_{1}$ from its initial node $v_{s}$ to its end node $v_{f}$ and the last visited edge is its back edge $e_{b}$. Each time that a new node on the path is visited, recurrence (1) is applied, obtaining: $\left(\alpha_{i}^{j}, \beta_{i}^{j}\right) \rightarrow\left(\alpha_{i+1}^{j}, \beta_{i+1}^{j}\right), j=$ $1, \ldots, 3$. When the search arrives to $v_{f}$, we have obtained the pairs $\left(\alpha_{f}^{1}, \beta_{f}^{1}\right)$, $\left(\alpha_{f}^{2}, \beta_{f}^{2}\right)$ and $\left(\alpha_{f}^{3}, \beta_{f}^{3}\right)$. The last edge processed is $e_{b}$ and for this, we use two temporal variables $\alpha_{C 1}$ and $\beta_{C 1}$ defined as: $\alpha_{C 1}=\alpha_{f}^{1}, \beta_{C 1}=\beta_{f}^{1}-\beta_{f}^{3}$ for the monotone case or according to the signs associated with $e_{b}$ this last rule is tuned. And, the numbers of models for the internal cycle $C_{1}$, is $\# S A T\left(C_{1}\right)=\alpha_{C 1}+\beta_{C 1}$.

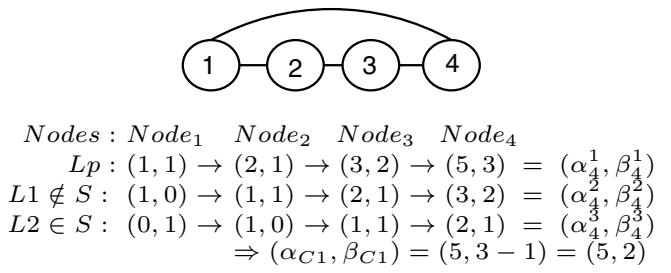

Fig. 7. Processing the most internal cycle

\section{Case 2: Processing an External Embedded Cycle}

Let $C_{j}=C_{i+1}$ be the following external embedded cycle of the last processed cycle $C_{i}$. After processing an internal embedded cycle $C_{i}$, all the cycle is contracted into a single node $C r_{s f}$ where $s$ is the number of the initial node and $f$ is the number of the final node of the path in $C_{i}$. Then, $C r_{i j}$ is now a new fat node on the path formed by the nodes: $\left(V\left(C_{j}\right)-V\left(C_{i}\right)\right) \cup C r_{i j}$.

We use new three computing threads for processing the external cycle $C_{j}$ according to the previous case $1 . C_{j}$ is traversing as a path and appliying recurrence (1) over each node of the path until arrive to the fat node $C r_{s f}$. When the computing threads cross by $C r_{s f}$, each current pair $\left(\alpha_{x}^{i}, \beta_{x}^{i}\right), i=1,2,3$ is updated according to the following recurrence.

$$
\begin{array}{r}
\alpha_{x+1}^{i}=\alpha_{f}^{2} \cdot \alpha_{x}^{i}+\alpha_{f}^{3} \cdot \beta_{x}^{i} \\
\beta_{x+1}^{i}=\beta_{f}^{2} \cdot \alpha_{x}^{i}+\beta_{f}^{3} \cdot \beta_{x}^{i}, \text { for } i=1,2,3 .
\end{array}
$$

Obtaining the new pairs $\left(\alpha_{x+1}^{i}, \beta_{x+1}^{i}\right)$, for $i=1,2,3$. We will denote the application of the recurrence ( 3) as $\left(\alpha_{x}, \beta_{x}\right) \odot\left(\alpha_{x+1}, \beta_{x+1}\right)$. As it exists an implicit back edge into the contracted fat node $C r_{s f}$ then we have to update the pair $\left(\alpha_{x+1}^{1}, \beta_{x+1}^{1}\right)$ as $\left(\alpha_{x+1}^{1}, \beta_{x+1}^{1}\right)=\left(\alpha_{x+1}^{1}, \beta_{x+1}^{1}-\beta_{x}^{1} * \beta_{f}^{3}\right)$. We will denote the processing of a back edge by $\hookleftarrow$, then $\left(\alpha_{x}, \beta_{x}\right) \hookleftarrow\left(\alpha_{x+1}, \beta_{x+1}\right)$ meaning the application of the formula $\left(\alpha_{x+1}, \beta_{x+1}\right)=\left(\alpha_{x+1}, \beta_{x+1}-\beta_{x} * \beta_{f}^{2}\right)$.

We obtain new current values for the last node of the cycle $C_{j}:\left(\alpha_{f}^{1}, \beta_{f}^{1}\right),\left(\alpha_{f}^{2}, \beta_{f}^{2}\right)$, $\left(\alpha_{f}^{3}, \beta_{f}^{3}\right)$ and the cycle $C_{j}$ is contracted into a new fat node $C_{k f}$ where $k$ was the number of the initial node and $f$ was the number of the final node processed in 
$C_{j}$. In this way, we process any embedded cycle until arrives to the most external cycle of $G_{F}$.

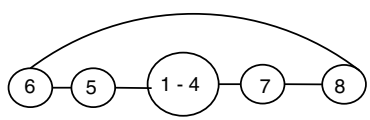

$$
\begin{aligned}
& G_{2} \text { Node }_{6} \text { Node }_{5} \quad \text { Node }_{1-4} \quad \text { Node }_{7} \quad \text { Node }_{8} \\
& L p:(1,1) \rightarrow(2,1) \rightarrow(3,2) \odot(13,8) \hookleftarrow(13,6) \rightarrow(19,13) \rightarrow(32,19)=\left(\alpha_{8}^{1}, \beta_{8}^{1}\right) \\
& L 1 \notin S:(1,0) \rightarrow(1,1) \rightarrow(2,1) \odot(8,5) \hookleftarrow(8,4) \rightarrow(12,8) \rightarrow(20,12)=\left(\alpha_{8}^{2}, \beta_{8}^{2}\right) \\
& L 2 \in S:(0,1) \rightarrow(1,0) \rightarrow(1,1) \odot(5,3) \hookleftarrow(5,2) \rightarrow(7,5) \rightarrow(12,7)=\left(\alpha_{8}^{3}, \beta_{8}^{3}\right) \\
& \Rightarrow(32,19)-(0,7)=(32,12)
\end{aligned}
$$

Fig. 8. Processing an external embedded cycle

Notice that our proposal for counting models on a set $D$ of embedded cycles has a linear time complexity over the number of edges $|E(D)|$. Then, we have shown that for some restricted cases of a $2-\mathrm{CF} F$, particularly when $G_{F}$ contains only independent and embedded cycles, \#2-SAT $(F)$ can be computed in polynomial time.

\section{Conclusion}

\#SAT problem for the class of Boolean formulas in 2-CF is a classical \# $\mathrm{P}$ complete problem. However, However, there are several instances of 2-CF's for which \#2SAT can be solved efficiently.

We have shown here, different polynomial-time procedures for counting models of Boolean formulas for subclasses of 2-CF's. For example, for formulas whose contrained graph is acyclic, its corresponding number of models is computed in linear time.

Given a formula $F$ in 2-CF, we show that if the cycles in its constrained graph $G_{F}$ can be arranged as independent and embedded cycles, then we can count efficiently the number of models of $F$.

Thus, the unique graph topology for the constrained graph $G_{F}$ of a $2-\mathrm{CF} F$ where the computation of \#2SAT $(F)$ continues being intractable is when $G_{F}$ has intersected cycles and they can not be arranged as embedded cycles.

\section{References}

1. Angelsmark, O., Jonsson, P.: Improved Algorithms for Counting Solutions in Constraint Satisfaction Problems. In: Rossi, F. (ed.) CP 2003. LNCS, vol. 2833, pp. 81-95. Springer, Heidelberg (2003)

2. Dahllöf, V., Jonsonn, P., Wahlström, M.: Counting models for 2SAT and 3SAT formulae. J. Theoretical Computer Sciences 332(1-3), 265-291 (2005)

3. Darwiche, A.: On the Tractability of Counting Theory Models and its Application to Belief Revision and Truth Maintenance. J. of Applied Non-classical Logics 11(1-2), 11-34 (2001) 
4. De Ita, G., Tovar, M.: Applying Counting Models of Boolean Formulas to Propositional Inference. J. Advances in Computer Science and Engineering Researching in Computing Science 19, 159-170 (2006)

5. De Ita, G., Bello, P., Contreras, M.: New Polynomial Classes for \#2SAT Established via Graph-Topological Structure. Engineering Letters 15(2), 250-258 (2007)

6. Dubois, O.: Counting the number of solutions for instances of satisfiability. J. Theoretical Computer Sciences 81(1), 49-64 (1991)

7. Fürer, M., Prasad, S.K.: Algorithms for Counting 2-SAT Solutions and Coloring with Applications. Technical Report No. 33, Electronic Colloqium on Comp. Complexity (2005)

8. Littman, M.L., Pitassi, T., Impagliazzo, R.: On the Complexity of counting satisfying assignments. Technical Report Unpublished manuscript

9. Roth, D.: On the hardness of approximate reasoning. J. Artificial Intelligence 82, 273-302 (1996)

10. Russ, B.: Randomized Algorithms: Approximation, Generation, and Counting, Distingished dissertations. Springer (2001)

11. Zhang, W.: Number of models and satisfiability of set of clauses. J. Theoretical Computer Sciences 155(1), 277-288 (1996) 